\title{
Théologiques
}

Théologiques

\section{Le zen et le soi}

\section{Victor Sogen Hori}

Volume 12, numéro 1-2, 2004

Le Soi dans tous ses états

URI : https://id.erudit.org/iderudit/011558ar

DOI : https://doi.org/10.7202/011558ar

Aller au sommaire du numéro

Éditeur(s)

Faculté de théologie et de sciences des religions, Université de Montréal

ISSN

1188-7109 (imprimé)

1492-1413 (numérique)

Découvrir la revue

Citer cet article

Sogen Hori, V. (2004). Le zen et le soi. Théologiques, 12(1-2), 125-133.

https://doi.org/10.7202/011558ar

Tous droits réservés $\odot$ Faculté de théologie et de sciences des religions, Université de Montréal, 2005
Ce document est protégé par la loi sur le droit d'auteur. L'utilisation des services d'Érudit (y compris la reproduction) est assujettie à sa politique d'utilisation que vous pouvez consulter en ligne.

https://apropos.erudit.org/fr/usagers/politique-dutilisation/
Cet article est diffusé et préservé par Érudit.

Érudit est un consortium interuniversitaire sans but lucratif composé de l’Université de Montréal, l'Université Laval et l'Université du Québec à Montréal. Il a pour mission la promotion et la valorisation de la recherche. https://www.erudit.org/fr/ 


\title{
Le zen et le soi $^{1}$
}

\author{
Victor Sōgen HoRI \\ Faculté d'études religieuses \\ Université McGill
}

Les contributions des autres intervenants dans le débat sur le soi, particulièrement celles des scientifiques, se révèlent extrêmement intéressantes par la façon dont elles mettent en question nos notions conventionnelles du soi. Le colloque "Le Soi dans tous ses états» et le présent numéro en témoignent. Je suis donc honoré de voir ma présentation associée aux leurs. À la différence des autres collaborateurs à ce numéro cependant, je n'expose pas un compte-rendu technique de mes recherches en science des religions. Les responsables de cette publication m'ont plutôt demandé de rendre compte de mon expérience personnelle dans la méditation bouddhiste et dans la pratique monastique.

Après avoir complété mes études doctorales à l'Université Stanford, je suis allé au Japon en 1970 pour étudier la philosophie japonaise à l'Université de Kyoto. Durant mon séjour là-bas, j’ai développé un profond intérêt pour la pratique de la méditation zen, au point de vouloir m’y engager entièrement. En conséquence, en 1976, après avoir soumis ma thèse doctorale, j'ai fait la demande au temple Daitoku-ji (de l'école zen Rinzai), à Kyoto, en vue d'être ordonné moine bouddhiste zen. L'année suivante, en 1977, je suis entré dans le monastère zen de Daitoku-ji et, pendant les treize années qui ont suivi, je me suis engagé totalement dans le zen kōan et la pratique de la méditation. En 1990, je suis revenu au Canada et j'ai repris la vie d'un universitaire. Aussi, je voudrais dire quelques mots au sujet du soi en relation avec la pratique zen.

1. Texte d'une communication faite au colloque «Le Soi dans tous ses états » (Montréal, 18-19 septembre 2003). Traduit de l'anglais par Dany Rodier et révisé par Alain Gignac. 


\section{La non-dualité du soi et de l'autre}

Le zen est une branche du bouddhisme Mahāyāna et enseigne, par conséquent, la doctrine bouddhiste traditionnelle du non-soi. À la différence de la plupart des autres religions, qui soutiennent l'existence d'un soi éternel, le bouddhisme enseigne que même s'il paraît y avoir un soi qui agit dans l'expérience quotidienne, ce soi est seulement provisoire et n'a aucune substance permanente. Fondamentalement - c'est-à-dire au niveau fondamental, non au niveau expérientiel - il n'y a pas de soi. Beaucoup de ceux qui sont informés de cet enseignement bouddhiste sont incapables d'imaginer ce que le non-soi peut possiblement signifier. Bien plus, ils sont incapables de croire qu'une religion organisée puisse tenir un tel enseignement. Je ne veux pas ici essayer d'expliquer cette notion, car cela prendrait beaucoup trop d'espace. Plutôt, je voudrais procéder en discutant directement de la manière dont le zen traite du sujet du non-soi.

Comme je l'ai dit, le zen est une branche du bouddhisme Mahāyāna. L'enseignement dominant du bouddhisme Mahāyāna est celui du vide. La doctrine du vide est, elle aussi, une doctrine complexe, mais un de ses aspects est l'idée selon laquelle les catégories dualistes, dont la pensée se sert tous les jours, sont vides. La plupart des jugements usuels que l'on fait dans une journée sont dualistes. Un jugement comme "Oh! Il est tard, je dois me dépêcher " présuppose une dichotomie entre le tard et le tôt; "Cette vieille voiture tombe en ruine", entre le vieux et le nouveau; "Il est un bon enseignant", entre le bon et le mauvais; "C'est une aubaine ", entre le bon marché et le cher. Le bouddhisme enseigne que tous les jugements de ce genre sont posés à partir du point de vue d'un soi. Ce qui est tard pour une personne est tôt pour une autre, ce qui est vieux pour l'une est nouveau pour l'autre, etc. Ces distinctions dichotomiques — tard/tôt, vieux/nouveau, bon/mauvais, bon marché/cher — ne sont pas absolues; elles sont vides.

Souvent, le bouddhisme Mahāyāna exprime le vide de ces catégories en créant des contradictions: le tard est tôt, le vieux est nouveau, le bon est mauvais, le bon marché est cher. Des propositions contradictoires comme celles-ci sont des signes du vide des catégories dualistes. Une pente va vers le haut pour celui qui se tient à sa base et va vers le bas pour celui qui se tient à son sommet. Le haut est l'opposé du bas, mais toute pente va à la fois vers le haut et vers le bas. La pente en soi ne va 
ni vers le haut ni vers le bas. Elle est, c'est tout. Pour la pente elle-même, le haut est le bas.

Certaines paires dualistes ne sont pas si manifestement relatives, si manifestement vides. Considérons la paire soi/autre. La plupart des gens peuvent comprendre ce que cela signifie de dire que le vieux est nouveau et que le bon est mauvais, car ceci peut être expliqué en disant que ce qui est vieux pour une personne est nouveau pour une autre, ce qui est bon pour une personne est mauvais pour une autre. Il y a un troisième terme - la personne qui pose le jugement - entre les deux termes opposés, vieux et nouveau, bon et mauvais. Mais que faire de la paire soi/autre, où la personne posant le jugement — le soi — est elle-même l'un des termes à propos desquels le jugement est posé? Ici, le bouddhisme Mahāyāna, sous sa forme zen, propose une profonde réflexion. Même le soi et l'autre sont non-duaux. Le soi est l'autre et l'autre est le soi.

\section{La méditation zen à partir du koan}

Comment le zen enseigne-t-il la non-dualité du soi et de l'autre? Ici, le zen se distancie considérablement du bouddhisme traditionnel quant à la pratique de la méditation. Dans le bouddhisme traditionnel, il y a deux types de pratique méditative, samatha et vipassana. Samatha est habituellement traduit par "calme, tranquillité " et vipassana, par "vision pénétrante» (proche de l'anglais insight). L'instruction sur la méditation débute généralement par samatha, ou la méditation du calme. L'enseignant instruit l'élève pour qu'il concentre son attention sur un seul objet, la respiration par exemple. Pour conserver l'attention sur la respiration, l'enseignant apprend à l'élève à compter - un, deux, trois, etc., jusqu'à dix - à chaque expiration et à répéter ce décompte encore et encore. On peut aussi opter pour d'autres méthodes. L'enseignant peut instruire l'élève à concentrer son attention sur une sensation éprouvée dans une partie de son corps ou sur une image (par exemple, un cercle). Quand la pensée n'est pas remplie de sa confusion et de son agitation habituelles, elle commence peu à peu à s'éclaircir. La pensée, dit-on, est comme un verre d'eau trouble. Si le verre demeure longtemps immobile, les sédiments descendent jusqu'au fond et l'eau devient graduellement transparente. Quand l'élève a atteint une certaine mesure de tranquillité et de clarté d'esprit, l'enseignant débute alors la formation sur la méditation vipassana. Dans cette dernière, l'élève apprend les concepts du bouddhisme 
— impermanence, souffrance, non-soi, etc. — et il analyse son expérience à travers ces concepts. Cette vision pénétrante vipassana de la nature de l'expérience, pense-t-on, n'est pas possible tant que la pensée n'a pas d'abord atteint le calme samatha.

La méditation zen, fondée sur le kōan, est très différente. Un kōan est un problème paradoxal que l'enseignant donne à l'élève comme objet sur lequel se fixe l'attention dans la méditation. Un célèbre kōan utilisé avec les débutants pourrait s'énoncer ainsi: "De deux mains on produit un son (par applaudissement); quel est le son d'une seule main?» Un autre est: "Quel est ton visage original avant que ton père et ta mère naissent? » La question n'est pas "Quel est ton visage original avant que tu naisses ? ", mais bien "avant que ton père et ta mère naissent? ". Ce type de questions ne peut pas être répondu rationnellement. Alors que celui qui médite réfléchit à des questions de ce genre, il risque des hypothèses à tout hasard ou essaie de les interpréter symboliquement. Au moins une fois par jour, il doit rencontrer le maître zen qui lui demande s'il est parvenu à comprendre. Comme l'élève devient de plus en plus troublé, il en vient aussi à douter de plus en plus de lui-même, tandis que le maître zen, de son côté, se fait de plus en plus exigeant. Et plutôt que d'atteindre le calme et la tranquillité de la méditation samatha, l'élève zen devient de plus en plus confus intellectuellement, de plus en plus agité émotionnellement.

Au moment où il lutte avec le kōan, l'élève zen ne comprend pas la nature de la question à laquelle il doit répondre. Il pense que la question "Tu connais le son que produisent deux mains (par applaudissement); quel est le son d'une seule main?" se rapporte aux mains. Il pense que la question "Quel est ton visage original avant que ton père et ta mère naissent ? " se rapporte au visage. Ce n'est pas le cas. Elles se rapportent au deux et à l'un, à la non-dualité. "Tu connais le son que produisent deux mains" signifie: "Tu connais ce qu'est la dualité du soi et de l'autre». "Quel est le son d'une seule main?" revient à demander: "Quelle est la non-dualité du soi et de l'autre? " La mère et le père sont le yin et le yang dans la pensée chinoise. Ici, ils représentent le soi et l'autre. "Quel est ton visage original avant que ton père et ta mère naissent ? ", c'est demander "Avant que la dualité du soi et de l'autre naisse, quelle est la non-dualité du soi et de l'autre?».

Bien qu'il soit possible d'interpréter ces questions kōan de façon métaphorique, il n'est cependant pas possible d'y répondre ainsi. On ne 
peut pas comprendre la non-dualité du soi et de l'autre au moyen de la pensée intellectuelle, car dans ce processus il y a un sujet de la pensée et un objet de la pensée, un soi qui pense et un objet à propos duquel le soi pense. La pensée intellectuelle est fondamentalement dualiste et intrinsèquement incapable de saisir la non-dualité. La pensée intellectuelle peut bien étiqueter son objet comme «non-dual», son acte même de penser rend son objet dual. C'est comme estampiller le mot "vierge» sur une feuille de papier vierge; l'acte même d'estampiller «vierge» fait en sorte que la feuille n'est plus vierge. En conséquence, pour vraiment comprendre la non-dualité du soi et de l'autre, on ne peut la penser intellectuellement. On doit l'expérimenter. On doit soi-même devenir la non-dualité du soi et de l'autre. Comment cela se produit-il ?

\section{Le son d'une seule main}

Un moine zen se répète constamment et se pose à lui-même la question du kōan: "Quel est le son d'une seule main?» De prime abord, le moine s'attendait à ce que la réponse au kōan apparaisse un jour devant lui comme la solution à une énigme. Dit autrement, il pensait qu'il s'agirait d'un objet de la conscience, d'un objet de la vue. C'est ce à quoi on pourrait s'attendre si on essayait de comprendre le kōan intellectuellement. Cependant, la constante répétition du kōan imprime ce dernier dans la conscience, de sorte que le kōan n'est plus simplement un objet de la vue, mais colore la vue elle-même. Éventuellement, sans aucun effort conscient, le kōan du «son d'une seule main » s'élève continuellement à la conscience, se répétant lui-même sans cesse chaque fois que l'attention n'est pas fixée sur autre chose. Il s'agit d'une des premières étapes que l'on peut reconnaître dans le processus qui conduit à devenir «un» avec le kōan. "Le son d'une seule main» a tellement envahi la conscience de l'élève qu'il n'est dorénavant plus l'objet de l'attention dans la conscience, mais forme l'arrière-plan de tout autre objet de l'attention.

Finalement, il vient un temps où le moine «réalise ${ }^{2}$ » que sa propre recherche d'une réponse au kōan et la façon dont lui-même réagit à son

2. Note des traducteurs : l'auteur joue sur la double acception du mot anglais realize, comme il deviendra évident plus loin : faire et prendre conscience. Nous l'avons donc rendu tel quel en français, avec les guillemets, puisque réaliser ne supporte que la premier sens anglais. 
incapacité à pénétrer le kōan sont elles-mêmes l'activité du kōan qui œuvre en lui. Il s'agit là d'un point difficile à expliquer. Le kōan n'est pas simplement une entité statique, quelque chose ayant une nature propre fixe et pouvant être appréhendé. C'est plutôt une activité, l'activité qui consiste à chercher à comprendre le kōan, lequel utilise le moine et sa pensée comme son arène. Le son d'une seule main est l'activité qui consiste à chercher une réponse au kōan, mais aussi la réponse qui constitue l'objet recherché. Le kōan est à la fois un objet de conscience et l'activité subjective de la conscience qui cherche à comprendre le kōan. Le moine lui-même, dans sa recherche, est le kōan. La «réalisation» de ceci consiste en la vision pénétrante, la réponse au kōan. D'abord, il y avait un sujet de conscience qui essayait de pénétrer un kōan, lequel était considéré simplement comme un objet de conscience. Sujet et objet - ce sont les deux mains de l'applaudissement. Quand le moine prend conscience que le kōan n'est plus simplement un objet de conscience, mais qu'il est lui-même le kōan en tant qu'activité de recherche de la réponse au kōan, alors le sujet et l'objet ne sont plus séparés et distincts. Le moine est devenu un avec le kōan, ou peut-être plus exactement, le kōan est devenu un avec lui - une seule main. Il «réalise » le kōan, dans les deux acceptions anglaises du mot "réaliser». D'une part, c'est une reconnaissance cognitive, mais, d'autre part, c'est aussi «rendre réel», du fait que la reconnaissance cognitive ne pouvait se produire tant que lui-même n'avait pas instauré l'unité du sujet avec l'objet ${ }^{3}$.

\section{Le soi dans tous ses états}

Le dualisme cartésien réapparaît un peu partout dans la pensée scientifique et philosophique occidentale, sous plusieurs formes, particulièrement celle de la dichotomie entre le sujet de la conscience et un objet de la conscience correspondant. Or, dans ce numéro, nous sommes exposés à une variété de conceptions du soi et plusieurs parmi elles remettent en question ce dualisme cartésien de la pensée et du corps, particulièrement la conception mise de l'avant par les neurosciences, qui n'est pas sans analogie avec le modèle zen du non-soi. Dans cette ligne, j'aimerais revenir sur une communication marquante du colloque «Le Soi dans tous ses

3. Ce passage sur «le son d'une seule main " reprend et adapte une partie de Hori 2000. 
états ", de la part du $\mathrm{D}^{\mathrm{r}}$ Michael Gazzaniga: "Seeking the Self in a Distributed Cortical Network ${ }^{4} »$.

La présentation du Dr Gazzaniga explique les effets du sectionnement du corps calleux entraînant l'isolement des hémisphères gauche et droit du cerveau, habituellement joints. Cette chirurgie fut pratiquée naguère sur des patients épileptiques dans l'espoir que cela soulagerait leurs crises. Après la chirurgie, les patients au cerveau divisé parurent d'abord tout à fait normaux, mais une enquête plus poussée montra de radicales anomalies.

À partir de l'étude de ces anomalies, l'on peut tirer des implications philosophiques qui mettent en question la notion traditionnelle d'une conscience de soi unitaire. Dans l'un des tests, on a montré à un patient au cerveau divisé (split brain) deux images simultanément, une image à chaque hémisphère. Une image d'une patte de poulet a été montrée à l'œil droit du patient (lequel transmet à l'hémisphère gauche) et une image d'une scène enneigée a été présentée à l'œil gauche (lequel transmet à l'hémisphère droit). On a ensuite montré au patient une variété d'autres images et on lui a demandé d'indiquer, en pointant avec sa main, une image liée à l'image originale. Il a correctement choisi avec sa main droite (contrôlée par son hémisphère gauche) une image d'un poulet pour aller avec l'image de la griffe de poulet, qu'il a vue avec l'œil droit. Et il a aussi correctement choisi avec sa main gauche (contrôlée par son hémisphère droit) une image d'une pelle pour aller avec l'image originale de la scène enneigée vue par l'œil gauche. Mais quand on l'a interrogé sur son choix des images, le patient était tout à fait conscient d'avoir choisi une image d'un poulet pour aller avec l'image originale d'une patte de poulet, mais il était inconscient d'avoir vu l'image de la scène enneigée, même si sa main gauche avait fait le correct choix d'une pelle à neige pour aller avec elle. Il était tout à fait conscient de ce que son œil droit avait vu et de ce que sa main droite avait choisi, mais il était inconscient de ce que son côté gauche avait vu et fait.

Des résultats d'expériences comme ceux-ci semblent indiquer que le soi n'est pas une entité unitaire, qu'il y a des activités fragmentaires du

4. N.D.L.R. La communication de Michael S. Gazzaniga n'a pu être publiée dans ce numéro. Toutefois, il est question des travaux de ce chercheur dans l'article de JeanNoël Missa. 
soi qui peuvent faire des choix intelligents en réponse à des problèmes posés, mais qui sont étrangers à la conscience. Même si l'on ne devrait pas généraliser sans avoir d'abord plus d'évidences, cet exemple semble avoir de larges implications. Il semble indiquer que, dans un réseau cortical dispersé, le soi est peut-être lui-même fragmenté et dispersé, en contraste avec la notion traditionnelle du soi en Occident, laquelle dépeint le soi comme une entité unitaire, le sujet de la conscience (par ailleurs en relation de dualité avec l'objet corporel).

La conception zen du soi met en question le dualisme cartésien de la pensée et du corps à sa racine même. Celui-ci présuppose que la pensée est une substance, que le corps en est une autre, et qu'il y a deux et seulement deux substances de ce genre. Or, l'expérience zen, métaphoriquement décrite comme "le son d'une seule main", "le visage original avant que père et mère naissent ", réfère à la "réalisation » que le sujet de l'expérience et l'objet de l'expérience ne sont pas deux. Ils ne sont pas des substances - entités permanentes et essentielles — et il n'y en a pas deux. Bien sûr, il y a un soi apparent, bougeant et interagissant dans le monde phénoménal, apparaissant sous diverses formes, agissant de différentes façons. Mais au niveau fondamental, il n'y a pas de soi substantiel, essentiellement séparé du monde à l'intérieur duquel il paraît bouger. Fondamentalement, il n'y a pas de soi. Il n'y a que tous ses états. 


\title{
Référence
}

Hori, V.S. (2000), «Kenshō and Kōan in the Rinzai Zen Curriculum», dans S. Heine et D.S. Wright, dir., The Kōan: Texts and Contexts in Zen Buddhism, Oxford, Oxford University Press, p. 280-315.

\section{Résumé}

À partir de son expérience, l'auteur montre que la pratique méditative zen, par l'utilisation de kōan (énigmes), entend dépasser la dualité phénoménale, puisque le kōan cesse d'être l'objet de l'attention pour devenir une activité qui travaille celui qui médite. La vision bouddhiste de non-soi remet en question le dualisme cartésien encore si influent en Occident — rejoignant ainsi certaines observations expérimentales des neurosciences.

\begin{abstract}
From his own, personal experience, the author shows that using riddles (kōan), in the practice of Zen meditation, serves to move one beyond a dualistic reality, since the kōan ceases to be the object of attention and becomes an activity which works on the one who meditates. The Buddhist vision of non-self brings into question the Cartesian dualism that is still so prevalent in the West, thus reuniting certain experimental observations from the field of neurosciences.
\end{abstract}

(C) Revue Théologiques 2004. Tout droit réservé. 